\title{
LISTADO DE ORIBÁTIDOS (ACARI, ORIBATIDA) DE TÚNEZ
}

\author{
L. S. Subías \& U. Ya. Shtanchaeva \\ Departamento de Zoología y Antropología Física. Facultad de Biología. Universidad Complutense. 28040 Madrid. España. \\ E-mail: subias@bio.ucm.es; umukusum@mail.ru
}

\section{RESUMEN}

Se han estudiado los ácaros oribátidos de una serie de muestras de suelo de Túnez y se han identificado 113 especies, 95 de las cuales se citan por primera vez en Túnez. Se ha elaborado un listado sistemático en el que se incluyen las 136 especies conocidas hasta el momento y su distribución geográfica. La mayoría, 77 (56 \%), presentan características típicamente mediterráneas, y 16 se citan por primera vez en el norte de África.

Palabras clave: Ácaros oribátidos; Túnez; Listado sistemático; Nuevas citas; Distribución geográfica.

\section{ABSTRACT}

\section{Oribatid mites (Acari, Oribatida) from Tunisia}

The oribatid mites of several Tunisian soil samples were studied. 113 species were obtained, 95 of which are recorded for the first time from Tunisia. A systematic check-list with the 136 species known up to now is included, and their geographical distribution is given. Most of them, 77 (56\%), have a typical Mediterranean distribution and 16 are recorded for the first time from North Africa.

Key words: Oribatid mites; Tunisia; Systematic checklist; new records; Geographical distribution.

Recibido/Received: 14/04/2015; Aceptado/Accepted: 25/06/2015; Publicado en línea/Published online: 19/08/2015

Cómo citar este artículo/Citation: Subías, L. S. \& Shtanchaeva, U. Ya. 2015. Listado de oribátidos (Acari, Oribatida) de Túnez. Grael/sia, 71(2): e028. http://dx.doi.org/10.3989/graellsia.2015.v71.136

Copyright: (C) 2015 SAM y CSIC. Salvo indicación contraria, todos los contenidos de la edición electrónica de Grael/sia se distribuyen bajo licencia de uso y distribución Creative Commons Reconocimiento no Comercial 3.0. España (cc-by-nc).

\section{Introduction}

El número de especies de ácaros oribátidos conocido en la actualidad sobrepasa las 10.000 especies, según Subías [2004 (2014)], lo que contrasta con el muy escaso conocimiento de la fauna de oribátidos de Túnez que se tiene en la actualidad ya que sólo se conocían 38 especies, número que todavía es más significativo si se compara con los cerca del millar de especies de oribátidos conocidos de España, como ponen de manifiesto Subías et al. [2013 (2014)]. Con este estudio se contribuye además de forma significativa al conocimiento de los oribátidos del Mediterráneo occidental que, aunque según Subías \& Gil-Martín (1997) incluía 982 especies, sobrepasa claramente el millar en la actualidad. Es de destacar que estos autores separan la subregión Macaronésica de la del Norte de Africa, en cambio Niedbała (2012a) incluye las islas Canarias y Madeira en el Norte de África.

Túnez es la peor conocida de las tres naciones que componen el Magreb en lo que respecta a su fauna de ácaros oribátidos. En cambio Doumandji et al. (1985) publican un catálogo de los ácaros terrestres de Argelia, mientras que Subías et al. (1994) elaboran un listado de los oribátidos de Marruecos y Sahara occidental. Las primeras citas de oribátidos de Túnez se deben a André (1923) y no sería hasta 60 años después cuando Mahunka (1980) cita y describe varias especies nuevas que incrementan dicho conocimiento. Con posterioridad, Bernini \& Avanzati (1988, 1989a, 1989b, 1989c), Bernini et al. (1989) y Niedbała (2012b) realizan alguna cita más, 
pero es con el presente estudio cuando se empieza a tener un cierto conocimiento de dicha fauna.

\section{Material y métodos}

Las muestras de suelo del presente estudio han sido recolectadas por Zaballos, Pérez-González y Ghannem entre los días 15 y 19 de enero de 2013 en medios endogeos en prados y eriales de ribera y estepa; la tierra es recogida en profundidad y su mesofauna ha sido extraída por el sistema de lavado, quedando adherida en los papeles de filtro y separándose posteriormente los ácaros oribátidos a pincel en la lupa binocular y tras someterles a un aclarado con ácido láctico al $70 \%$ se ha procedido a su observación e identificación al microscopio. A la finalización de los análisis, el material se depositará en el Departamento de Zoología de la Facultad de Biología de la Universidad Complutense de Madrid.

Las muestras estudiadas proceden de las siguientes localidades (Fig. 1):

Muestra 1: Metline. 15-1-2013. 37 $11^{\prime} 34.67^{\prime \prime} \mathrm{N} 10^{\circ} 2^{\prime} 10.59^{\prime \prime} \mathrm{E}$. Zona de cultivo-pastizal, con asfódelos, cardos, etc. Encinas dispersas a unos $200 \mathrm{~m}$.

Muestra 2: $\quad$ Ras Djabel olivar. 15-1-2013. 37 $11^{\prime} 48.82^{\prime \prime} \mathrm{N} 10^{\circ}$ $8^{\prime} 2.16^{\prime \prime}$ E. Olivar en ladera, con sembrado de trigo. Tamizado de suelo bajo raíces de asfódelo.

Muestra 3: Ras Djabel cardonal. 15-1-2013. $37^{\circ} 10^{\prime} 40.67^{\prime \prime} \mathrm{N}$ $10^{\circ} 7^{\prime} 0.41^{\prime \prime} \mathrm{E}$. Cultivo-cardonal con asfódelos y cardos en las isletas sin cultivar, en ladera (similar al hábitat de Pseudanillus pastorum).

Muestra 4: Reserva Natural lago Ischkeul. 16-1-2013. $37^{\circ}$ $7^{\prime} 10.07^{\prime \prime} \mathrm{N} 9{ }^{\circ} 40^{\prime} 26.91^{\prime \prime E}$. "Recodos" del lago, zona tipo pastizal inundable con muchos cardos, junto a faldas de laderas de la montaña, rocosas, con olivos y cardonal. Con piedras adecuadas. Muy buena zona para Carábidos. Se tomaron submuestras en llanura, junto a raíces de plantas tipo asfódelo y bajo piedras, y otra en ladera pedregosa.

Muestra 5: $\quad$ Sidi Salah Bougrabin (N lago Ischkeul). 16-1-2013. $37^{\circ} 10^{\prime} 52.35^{\prime \prime} \mathrm{N} 9^{\circ} 34^{\prime} 24.86^{\prime \prime} \mathrm{E}$. Olivar centenario con piedras grandes. Se tomaron submuestras (sacos)

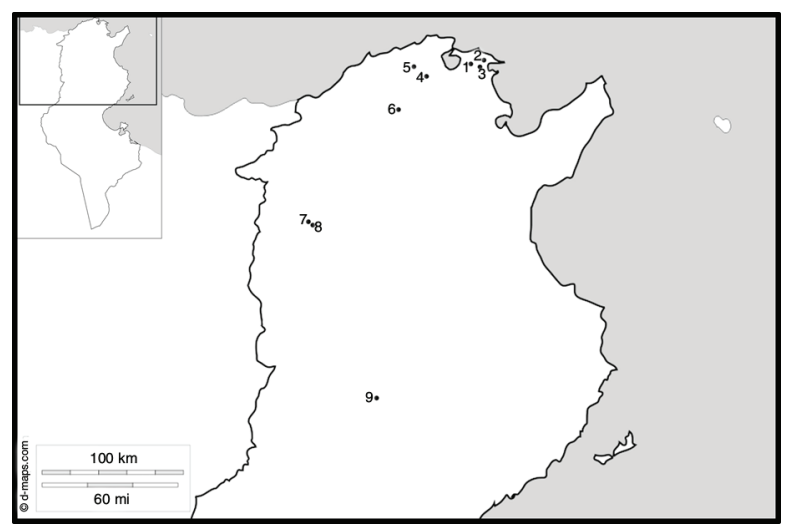

Fig. 1.- Mapa de Túnez con la localización de los puntos de muestreo.

Fig. 1.- Map of Tunisia with the location of sampling points. para lavar en talud y bajo olivos en ladera y junto a carretera.

Muestra 6: $\quad$ Sidi Nsir. 17-1-2013. 36 $54^{\prime} 12.61^{\prime \prime} \mathrm{N} 9^{\circ} 26^{\prime} 59.76^{\prime \prime} \mathrm{E}$ Pastizal-cardonal con asfódelo y piedras. Suelo bajo piedra.

Muestra 7: Le Keuf. 17-1-2013.36¹0'49.03”N 844'21.61"E. Paisaje mixto, fin de pinar, cardonales y cauces de arroyo. Terreno arcillo-margoso gris amarillento, muy seco. Bajo piedras del camino y talud, y bajo piedras en cauce de regato.

Muestra 8: Le Keuf (Este). 17-1-2013. 36 $6^{\circ} 43.97^{\prime \prime} \mathrm{N}$ $8^{\circ} 46^{\prime} 12.12^{\prime \prime}$. Cauce en campo de trigo. En talud con vegetación, junto a una liliácea.

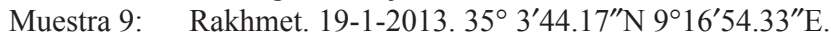
Olivar de cultivo, chumberas y crucíferas de cuneta. Bajo olivo. Con cierta humedad bajo la costra seca de los primeros centímetros de suelo.

\section{Resultados y Discusión}

Los resultados se presentan en el Apéndice, en el que se incluye el listado, ordenado sistemáticamente, de todos los oribátidos conocidos hasta ahora de Túnez y la distribución geográfica conocida actualmente de cada una de las especies, según Subías \& Shtanchaeva (2012). Las nuevas citas para el norte de África se indican con un asterisco $(*)$.

Se han recolectado un total de 113 especies de oribátidos de las que 95 son nuevas citas para la fauna de Túnez, lo que incrementa el número de especies conocidas en la actualidad a 136. Las especies citadas previamente, pero no encontradas en estos muestreos, no están marcadas con X en las distintas muestras estudiadas. Es de destacar que 16 de estas especies, señaladas con un *, se citan por primera vez en el norte de África destacando entre ellas Heptacarus supertrichus Piffl, 1967 que hasta ahora sólo se conocía de donde fue descrita, de Iraq, aunque con posterioridad fue también citada de México (Subías, 2004, 2014).

Por lo que respecta a la distribución geográfica de las especies listadas, la mayoría de ellas, 77 (lo que supone un $56 \%$ ) son de características mediterráneas, incluyendo entre ellas las de distribuciones paleárticas y holárticas meridionales o subtropicales; las restantes presentan, en general, amplias distribuciones: cosmopolitas, semicosmopolitas, holárticas, paleárticas o paleárticas occidentales, lo que concuerda con lo esperado ya que todo el territorio de Túnez se encuentra enclavado en la región Mediterránea. De los taxones descritos como nuevos por Mahunka $(1980,1987)$ sólo uno, Dissorhina ornata tunisica (Mahunka, 1980), permanece como endemismo ya que los demás han sido encontrados en otras localizaciones de la región del Mediterráneo occidental, fundamentalmente la península Ibérica.

\section{Agradecimientos}

Nuestro más sincero agradecimiento a los recolectores del material, Dr. Juan P. Zaballos y Sergio Pérez-González (Facultad de Biología, Universidad Complutense de Madrid) y a Samir Ghannèm (Facultad de Ciencias de Bizerte, Universidad de 
Cartago), especialistas en coleópteros endogeos, por poner a nuestra disposición las muestras de suelo objeto del presente estudio.

\section{Referencias}

André, M. M., 1923. Acariens recueillis in Tunisie (Le Kef) par M. le Dr. Larrousse dans des terriers de petits Rongeurs, (1re Liste). Bulletin du Museúm national d'Histoire naturelle, 9(7): 506-508.

Bernini, F. \& Avanzati, A. M., 1988. Taxonomic revision of Steganacarus (Steganacarus) magnus (Nicolet, 1855) (Acarida, Oribatida). Journal of Natural History, 22: 435-464. http://dx.doi.org/10.1080/00222938800770311

Bernini, F. \& Avanzati, A. M., 1989a. Notulae Oribatologicae XLVI. Steganacarus (Tropacarus) carinatus (C. L. Koch, 1841) and two new mediterranean Tropacarus species (Acarida, Oribatida). Acarologia, 30: 143-161.

Bernini, F. \& Avanzati, A. M., 1989b. Notulae Oribatologicae XLVIII. The taxonomic position of Steganacarus brevipilus (Berlese, 1923) and Tropacarus in the Steganacaridae system (Acarida, Oribatida). International Journal of Acarology, 15: 5-16.

Bernini, F. \& Avanzati, A. M., 1989c. Notulae Oribatologicae XLIX. Taxonomic revision of Steganacarus (Steganacarus) applicatus (Sellnick, 1920) and the description of a new west mediterranean Steganacarus species (Acarida, Oribatida). International Journal of Acarology, 15: 65-74.

Bernini, F., Bernini, S. \& Avanzati, A. M., 1989. Notulae oribatologicae XLII. Three new species belonging to the subgenus Tropacarus (Acari, Oribatida). Journal of African Zoology, 103: 155-177.

Doumandji, B., Boulfekhar, H., Guessoum, M. \& Niedbała, W., 1985. Catalogue del Acariens terrestres d'Algérie. Bulletin de Zoologie Agricole, $\mathrm{N}^{\circ}$ spéc.: 1-31.

Mahunka, S., 1980. Oribatids from Tunisian soils (Acari: Oribatida). I. Folia Entomologica Hungarica, 41(33): 123-134.
Mahunka, S., 1987. A survey of the family Carabodidae C. L. Koch, 1836 (Acari: Oribatida), II. Acta Zoologica Hungarica, 33(3-4): 399-434.

Niedbała, W., 2012a. Ptyctimous mites (Acari, Oribatida) in the fauna of North Africa, against the background of the Palaearctic fauna. Biological Letters, 49(2): 137-142. http://dx.doi.org/10.2478/v10120-012-0015-2

Niedbała, W., 2012b. Ptyctimous mites (Acari: Oribatida) of the Palaearctic Region. Distribution. Fauna Mundi, 5: $1-348$

Subías, L. S., 2004 (2014). Listado sistemático, sinonímico y biogeográfico de los ácaros oribátidos (Acariformes: Oribatida) del mundo (excepto fósiles). Graellsia, 60 (número extraordinario): 3-305. http://dx.doi.org/10.3989/ graellsia.2004.v60.iExtra [Actualización on-line de Febrero de 2014; http://escalera.bio.ucm.es/usuarios/ $\mathrm{bba} /$ cont/docs/RO_1.pdf].

Subías, L. S., Arillo, A. \& Gil-Martín, J., 1994. Oribátidos de Marruecos y Sahara Occidental. II. Listado de especies (Acari, Oribatida). Boletín de la Real Sociedad española de Historia Natural (Sección Biológica), 91(1-4): 129-134.

Subías, L. S. \& Gil-Martín, J., 1997. Systematic and biogeographic check-list of Oribatids from Western Mediterranean (Acari, Oribatida). Annali del Museo Civico di Storia Naturale "G. Doria”, 91: 459-498.

Subías, L. S. \& Shtanchaeva, U. Ya., 2012. Listado sistemático, sinonímico y biogeográfico de los ácaros oribátidos (Acari: Oribatida) mediterráneos. Boletín de la Real Sociedad española de Historia Natural (Sección Biológica), 106: 5-92.

Subías, L. S., Shtanchaeva, U. Ya. \& Arillo A., 2013 (2014). Oribátidos (Acari, Oribatida) de España peninsular e Islas Baleares. Distribución. Sociedad Entomológica Aragonesa. Monografías electrónicas (S.E.A.), 5: 255 pp. [Actualización on-line de Julio de 2014: http://escalera. bio.ucm.es/usuarios/bba/cont/docs/RO_28.pdf]. 
Apéndice.- Oribátidos de Túnez y su distribución geográfica conocida.

Appendix.- Oribatid mites from Tunisia and known geographical distribution.

\begin{tabular}{llllllllllll}
\hline & \multicolumn{1}{c}{ Muestras estudiadas } & & \\
\cline { 2 - 6 } TÚNEZ & 1 & 2 & 3 & 4 & 5 & 6 & 7 & 8 & 9 & Distribución \\
\hline
\end{tabular}

Ctenacaridae Grandjean, 1954

Beklemishevia hispaniola Pérez-Íñigo, 1997

Ctenacarus araneola (Grandjean, 1932)

Aphelacaridae Grandjean, 1954

Aphelacarus acarinus (Berlese, 1910)

Parhypochthoniidae Grandjean, 1932

Parhypochthonius aphidinus Berlese, 1904

Brachychthoniidae Thor, 1934

Brachychthonius hauserorum (Mahunka, 1979)

Eobrachychthonius oudemansi (Berlese, 1910)

Poecilochthonius italicus (Berlese, 1910)

Sellnickochthonius chinonei Subías y Shtanchaeva, 2011

Cosmochthoniidae Grandjean, 1947

Cosmochthonius foliatus Subías, 1982

Cosmochthonius reticulatus Grandjean, 1947

Haplochthoniidae Hammen, 1959

Haplochthonius sanctaeluciae Bernini, 1973

Sphaerochthoniidae Grandjean, 1947

Sphaerochthonius pallidus Muñoz-Mingarro, 1987

Sphaerochthonius splendidus (Berlese, 1904)

Protoplophoridae Ewing, 1917

Protoplophora palpalis Berlese, 1910

Hypochthoniidae Berlese, 1910

Hypochthonius rufulus Koch, 1835

Lohmanniidae Berlese, 1916

Cryptacarus promecus Grandjean, 1950

*Heptacarus supertrichus Piffl, 1967

Lohmannia hungarorum Mahunka, 1980

Papillacarus pseudoaciculatus Mahunka, 1980

Epilohmanniidae Oudemans, 1923

Epilohmannia cylindrica s. str. (Berlese, 1904)

*Epilohmannia cylindrica minima Schuster, 1960

Epilohmannia heterotricha Mahunka, 1966

Epilohmannia styriaca Schuster, 1960

Euphthiracaridae Jacot, 1930

Acrotritia hyeroglyphica (Berlese, 1916)

Phthiracaridae Perty, 1841

Atropacarus platakisi (Mahunka, 1979)

Steganacarus (S.) carusoi Bernini y Avanzati, 1989

Steganacarus (S.) magnus (Nicolet, 1855)

Steganacarus (Tropacarus) boulfekhari Niedbała, 1986

Steganacarus (Tropacarus) brevipilus (Berlese, 1923)

Steganacarus (Tropacarus) callainii Bernini, S. Bernini y Avanzati,1989

Nothridae Berlese, 1896

Nothrus perezinigoi Mahunka, 1980 
Appendix 1.- (Continued)

\begin{tabular}{l} 
TÚNEZ \\
\hline Crotoniidae Thorell, 1876 \\
Camisia horrida (Hermann, 1804) \\
Hermanniellidae Grandjean, 1934 \\
Hermanniella granulata (Nicolet, 1855) \\
Neoliodidae Sellnick, 1928 \\
Neoliodes theleproctus (Hermann, 1804) \\
Platyliodes doderleini (Berlese, 1883) \\
Gymnodamaeidae Grandjean, 1954 \\
Adrodamaeus femoratus (Koch, 1839) \\
Arthrodamaeus bicristatus Subías, Arillo y J. Subías, 1997 \\
*Arthrodamaeus cereus Subias, Arillo y J. Subías, 1997 \\
Gymnodamaeus bicostatus (Koch, 1835)
\end{tabular}

Aleurodamaeidae Paschoal y Johnston, 1985

Aleurodamaeus setosus (Berlese, 1883)

Damaeidae Berlese, 1896

Damaeus (Spatiodamaeus) verticillipes (Nicolet, 1855)

Metabelbella interlamellaris Pérez-Íñigo, 1987

Ceratoppiidae Kunst, 1971

Ceratoppia bipilis (Hermann, 1804)

Gustaviidae Oudemans, 1900

Gustavia fusifer (Koch, 1841)

Liacaridae Sellnick, 1928

Liacarus coracinus (Koch, 1841)

Xenillidae Woolley e Higgins, 1966

Xenillus tegeocranus (Hermann, 1804)

Eremaeidae Oudemans, 1900

Eueremaeus brevifer (Mahunka, 1980)

Eueremaeus oblongus (Koch, 1835)

Ctenobelbidae Grandjean, 1965

${ }^{*}$ Ctenobelba csiszarae Mahunka, 1977

*Ctenobelba pseudomahnerti Subías y Shtanchaeva, 2013

Amerobelbidae Grandjean, 1961

Mongaillardia aeoliana (Bernini, 1979)

Damaeolidae Grandjean, 1965

Damaeolus asperatus (Berlese, 1904)

Fosseremus laciniatus (Berlese, 1905)

Oribellidae Kunst, 1971

Oribella pectinata (Michael, 1885)

Oppiidae Sellnick, 1937

Lasiobelba arcidiaconoae (Bernini, 1973)

Graptoppia paraanalis Subías y Rodríguez, 1985

*Javieroppia cervus Mínguez y Subías, 1986

Ramusella (R.) assimiloides Subías y Rodríguez, 1987

Ramusella (R.) puertomonttensis Hammer, 1962

Ramusella (R.) sengbuschi tokyoensis (Aoki, 1974)

Ramusella (Insculptoppia) anuncata Subías y Rodríguez, 1986

Ramusella (Insculptoppia) terricola Subías y Rodríguez, 1986
Muestras estudiadas

$\begin{array}{lllllllll}1 & 2 & 3 & 4 & 5 & 6 & 7 & 8 & 9\end{array} \quad$ Distribución

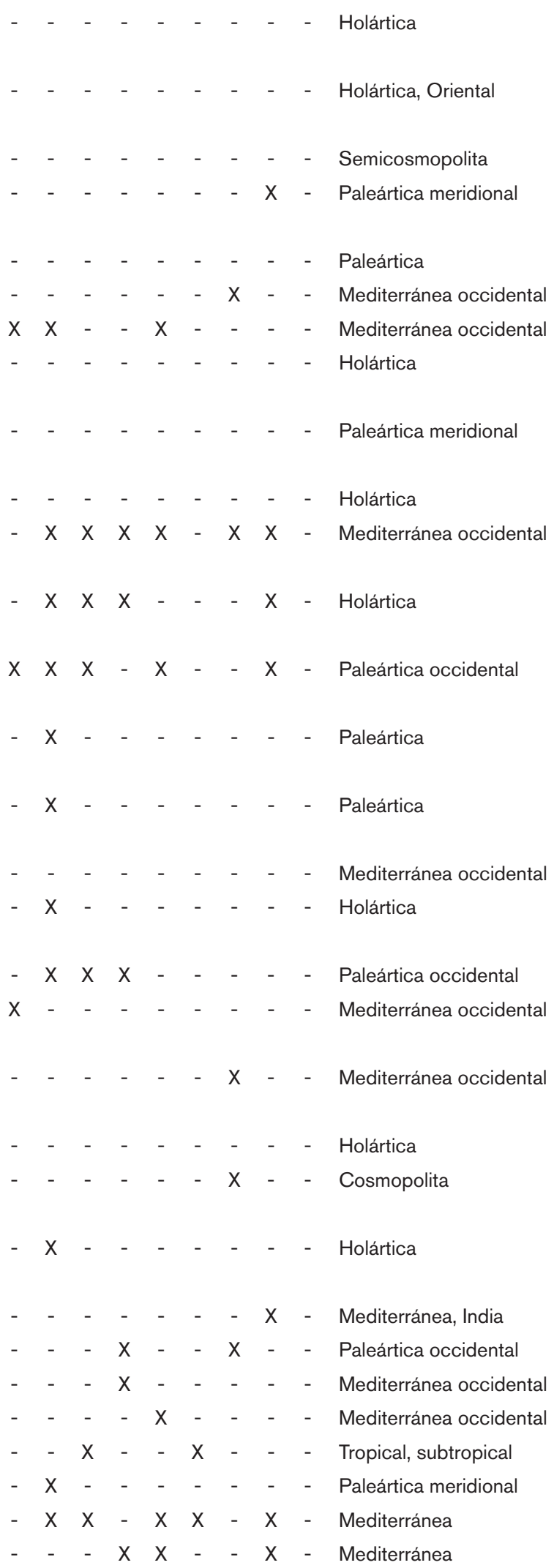


Appendix 1.- (Continued)

\begin{tabular}{|c|c|c|c|c|c|c|c|c|c|c|}
\hline \multirow[b]{2}{*}{ TÚNEZ } & \multicolumn{9}{|c|}{ Muestras estudiadas } & \multirow[b]{2}{*}{ Distribución } \\
\hline & 1 & 2 & 3 & 4 & 5 & 6 & 7 & 8 & 9 & \\
\hline Ramusella (Insculptoppiella) elongata (Paoli, 1908) & - & - & $\mathrm{x}$ & $x$ & $\mathrm{x}$ & - & $\mathrm{x}$ & $x$ & - & Mediterránea \\
\hline Ramusella (Rectoppia) strinatii (Mahunka, 1980) & - & $x$ & - & $x$ & - & - & $x$ & $x$ & - & Mediterránea \\
\hline Discoppia (Cylindroppia) cylindrica (Pérez-ĺñigo, 1965) & - & - & - & $x$ & - & - & $\mathrm{x}$ & $x$ & - & Paleártica, tropical \\
\hline Microppia minus s. str. (Paoli, 1908) & - & - & - & - & - & - & - & - & $\mathrm{x}$ & Cosmopolita \\
\hline Microppia minus longisetosa Subías y Rodríguez, 1988 & - & - & - & - & - & - & - & $\mathrm{x}$ & - & Paleártica meridional \\
\hline Rhinoppia obsoleta s. str. (Paoli, 1908) & - & - & - & $x$ & - & - & $\mathrm{x}$ & $x$ & - & Holártica, Australiana \\
\hline${ }^{*}$ Rhinoppia obsoleta curtiramosa Subías y Shtanchaeva, 2011 & $\mathrm{x}$ & $x$ & $\mathrm{x}$ & - & $\mathrm{x}$ & - & - & - & - & Mediterránea \\
\hline *Berniniella silvatica (Vasiliu y Călugăr, 1976) & - & $x$ & - & - & - & - & - & - & - & Mediterránea \\
\hline Dissorhina ornata s. str. (Oudemans, 1900) & - & - & - & - & - & - & $\mathrm{x}$ & $x$ & - & Holártica \\
\hline Dissorhina ornata tunisica (Mahunka, 1980) & - & - & - & - & - & - & - & - & - & Túnez \\
\hline *Neotrichoppia (Confinoppia) confinis (Paoli, 1908) & - & - & - & - & - & - & - & $\mathrm{x}$ & - & Paleártica occidental \\
\hline Oxyoppioides decipiens (Paoli, 1908) & $\mathrm{x}$ & - & - & - & - & - & - & - & - & Paleártica meridional \\
\hline \multicolumn{11}{|l|}{ Suctobelbidae Jacot, 1938} \\
\hline Suctobelbella (S.) acutidens pilososetosa Shtanchaeva y Subías, 2009 & - & $x$ & - & - & - & - & $\mathrm{x}$ & - & - & Mediterránea \\
\hline Suctobelbella (S.) italica (Mahunka, 1966) & - & $\mathrm{x}$ & - & - & - & - & - & $\mathrm{x}$ & - & Paleártica occidental \\
\hline Suctobelbella (Flagrosuctobelba) nasalis (Forsslund, 1941) & - & - & - & - & - & - & - & $\mathrm{x}$ & - & Paleártica \\
\hline \multicolumn{11}{|l|}{ Carabodidae Koch, 1837} \\
\hline Austrocarabodes arrogans Pérez-Íñigo, 1967 & $x$ & $x$ & - & - & - & - & - & $x$ & - & Mediterránea \\
\hline Odontocepheus villosus Mahunka, 1987 & - & - & - & - & - & - & - & - & - & Mediterránea occidental \\
\hline \multicolumn{11}{|l|}{ Tectocepheidae Grandjean, 1954} \\
\hline Tectocepheus velatus sarekensis Trägårdh, 1910 & - & $x$ & - & - & - & $x$ & $\mathrm{x}$ & $x$ & - & Cosmopolita \\
\hline \multicolumn{11}{|l|}{ Licneremaeidae Grandjean, 1931} \\
\hline Licneremaeus embeyisztini Mahunka, 1980 & - & - & - & - & - & - & - & - & - & Mediterránea occidental \\
\hline \multicolumn{11}{|l|}{ Scutoverticidae Grandjean, 1954} \\
\hline Scutovertex granulatus Mihelčič, 1957 & - & - & - & - & - & - & $x$ & - & - & Paleártica meridional \\
\hline Scutovertex minutus (Koch, 1836) & - & - & - & - & - & - & - & - & $x$ & Holártica \\
\hline Scutovertex sculptus Michael, 1879 & - & - & - & - & - & - & - & - & - & Paleártica \\
\hline \multicolumn{11}{|l|}{ Passalozetidae Grandjean, 1954} \\
\hline Bipassalozetes vicinus (Mihelčič, 1957) & - & $x$ & - & - & - & - & - & - & - & Paleártica, Kenia \\
\hline Passalozetes africanus Grandjean, 1932 & - & - & - & $x$ & $x$ & - & $x$ & $x$ & - & Paleártica centromerid. \\
\hline *Passalozetes onubensis Subías, Ruiz y Kahwash, 1990 & - & - & - & - & $x$ & - & - & - & - & Mediterránea occidental \\
\hline \multicolumn{11}{|l|}{ Phenopelopidae Petrunkevitch, 1955} \\
\hline Eupelops acromios (Hermann, 1804) & - & $x$ & - & - & $x$ & - & - & $x$ & - & Semicosmopolita \\
\hline Eupelops sulcatus (Oudemans, 1914) & - & - & - & - & - & - & $\mathrm{x}$ & $x$ & - & Holártica \\
\hline \multicolumn{11}{|l|}{ Microzetidae Grandjean, 1936} \\
\hline Berlesezetes ornatissimus (Berlese, 1913) & - & - & - & - & - & - & - & $x$ & - & Pantropical, subtropical \\
\hline \multicolumn{11}{|l|}{ Achipteriidae Thor, 1929} \\
\hline Achipteria coleoptrata (Linnaeus, 1758) & - & - & - & - & - & - & - & - & - & Holártica \\
\hline *Parachipteria magna (Sellnick, 1928) & $\mathrm{x}$ & $x$ & $\mathrm{x}$ & - & $x$ & - & - & - & - & Paleártica occidental \\
\hline \multicolumn{11}{|l|}{ Oribatellidae Jacot, 1925} \\
\hline Oribatella foliata Krivolutsky, 1974 & $x$ & $x$ & - & - & $x$ & - & - & - & - & Paleártica meridional \\
\hline \multicolumn{11}{|l|}{ Ceratozetidae Jacot, 1925} \\
\hline Ceratozetella maxima (Berlese, 1908) & - & $x$ & - & - & - & - & - & $x$ & - & Paleártica occidental \\
\hline Ceratozetes conjunctus Mihelčič, 1956 & - & - & - & - & - & - & - & $x$ & - & Mediterránea \\
\hline${ }^{*}$ Ceratozetes laticuspidatus Menke, 1964 & $x$ & $x$ & $x$ & $x$ & $x$ & - & $x$ & $x$ & - & Paleártica occidental \\
\hline Ceratozetes mediocris Berlese, 1908 & - & - & - & - & - & - & - & $x$ & - & Semicosmopolita \\
\hline${ }^{*}$ Ceratozetes peritus Grandjean, 1951 & - & - & - & - & $x$ & - & - & - & - & Holártica \\
\hline Euzetes globulus (Nicolet, 1855) & - & - & - & - & - & - & - & - & - & Paleártica, Argentina \\
\hline Trichoribates (Latilamellobates) incisellus (Kramer, 1897) & - & - & - & - & $\mathrm{x}$ & - & - & - & - & Holártica \\
\hline Zetomimus (Protozetomimus) acutirostris (Mihelčič, 1957) & - & - & - & $x$ & - & - & - & - & - & Mediterránea \\
\hline
\end{tabular}


Appendix 1.- (Continued)

\begin{tabular}{|c|c|c|c|c|c|c|c|c|c|c|}
\hline \multirow[b]{2}{*}{ TÚNEZ } & \multicolumn{9}{|c|}{ Muestras estudiadas } & \multirow[b]{2}{*}{ Distribución } \\
\hline & 1 & 2 & 3 & 4 & 5 & 6 & 7 & 8 & 9 & \\
\hline \multicolumn{11}{|l|}{ Chamobatidae Thor, 1937} \\
\hline${ }^{*}$ Chamobates birulai (Kulczynski, 1902) & $\mathrm{x}$ & $\mathrm{x}$ & - & - & $\mathrm{x}$ & - & - & - & - & Holártica \\
\hline Chamobates subglobulus (Oudemans, 1900) & - & - & - & - & - & - & - & - & - & Paleártica \\
\hline \multicolumn{11}{|l|}{ Punctoribatidae Thor, 1937} \\
\hline Minunthozetes quadriareatus Mínguez, Subías y Ruiz, 1986 & - & - & - & - & - & $x$ & - & - & - & Mediterránea occidental \\
\hline Minunthozetes tarmani Feider, Vasiliu y Călugăr, 1971 & - & $x$ & - & - & - & $x$ & - & - & - & Paleártica meridional \\
\hline${ }^{*}$ Punctoribates mundus Shaldybina, 1973 & - & - & $\mathrm{x}$ & $x$ & - & - & - & - & - & Paleártica meridional \\
\hline Punctoribates punctum (Koch, 1839) & $x$ & $x$ & $x$ & $\mathrm{x}$ & - & - & $x$ & $\mathrm{x}$ & - & Semicosmopolita \\
\hline \multicolumn{11}{|l|}{ Zetomotrichidae Grandjean, 1934} \\
\hline Zetomotrichus lacrimans Grandjean, 1934 & - & - & - & - & - & - & $\mathrm{x}$ & - & - & Mediterránea, tropical \\
\hline \multicolumn{11}{|l|}{ Mochlozetidae Grandjean, 1960} \\
\hline Podoribates longipes (Berlese, 1887) & - & $x$ & - & - & $x$ & - & - & - & - & Holártica \\
\hline \multicolumn{11}{|l|}{ Oribatulidae Thor, 1929} \\
\hline Lucoppia burrowsi (Michael, 1890) & $x$ & $x$ & $x$ & - & - & - & - & - & - & Holártica, tropical \\
\hline Oribatula (O.) tibialis (Nicolet, 1855) & - & - & - & - & - & - & - & - & - & Holártica \\
\hline Oribatula (Zygoribatula) arcuatissima Berlese, 1916 & - & - & - & - & $x$ & - & $\mathrm{x}$ & - & - & Holártica \\
\hline Oribatula (Zygoribatula) exarata Berlese, 1916 & - & - & $\mathrm{x}$ & - & $\mathrm{x}$ & - & $\mathrm{x}$ & $\mathrm{x}$ & - & Paleártica meridional \\
\hline Oribatula (Zygoribatula) glabra (Michael, 1890) & - & - & - & - & - & - & $x$ & - & - & Paleártica \\
\hline Oribatula (Zygoribatula) undulata Berlese, 1916 & - & - & - & - & $x$ & - & - & - & - & Pantropical, subtropical \\
\hline \multicolumn{11}{|l|}{ Hemileiidae J. y P. Balogh, 1984} \\
\hline Hemileius initialis (Berlese, 1908) & - & - & - & - & - & - & - & $x$ & - & Semicosmopolita \\
\hline \multicolumn{11}{|l|}{ Liebstadiidae J. y P. Balogh, 1984} \\
\hline Liebstadia pannonica (Willmann, 1951) & - & - & - & - & - & - & $\mathrm{x}$ & - & - & Holártica \\
\hline Liebstadia saifulmalukensis (Hammer, 1977) & - & - & - & - & $x$ & - & - & - & - & Paleártica meridional \\
\hline \multicolumn{11}{|l|}{ Scheloribatidae Grandjean, 1933} \\
\hline Scheloribates barbatulus Mihelčič, 1956 & - & - & - & - & - & - & - & $x$ & - & Paleártica meridional \\
\hline Scheloribates caprai Bernini, 1973 & $\mathrm{x}$ & - & - & - & - & - & - & - & - & Mediterránea occidental \\
\hline Scheloribates fimbriatus Thor, 1930 & - & $\mathrm{x}$ & - & - & - & - & $\mathrm{x}$ & $\mathrm{x}$ & - & Cosmopolita \\
\hline Scheloribates labyrinthicus Jeleva, 1962 & - & - & - & - & - & - & $x$ & $x$ & - & Mediterránea \\
\hline Scheloribates laevigatus s. str. (Koch, 1835) & $x$ & - & - & $x$ & $x$ & $\mathrm{x}$ & $\mathrm{x}$ & $x$ & - & Semicosmopolita \\
\hline *Scheloribates laevigatus angustirostris Mihelčič, 1957 & - & $\mathrm{x}$ & - & $\mathrm{x}$ & $\mathrm{X}$ & - & $\mathrm{x}$ & $\mathrm{x}$ & - & Paleártica occidental \\
\hline Scheloribates minifimbriatus Mínguez, Subías y Ruiz, 1986 & $x$ & $x$ & - & $\mathrm{x}$ & $x$ & - & $\mathrm{x}$ & $\mathrm{x}$ & - & Tropical, subtropical \\
\hline Topobates holsaticus Weigmann, 1969 & - & - & - & - & $x$ & - & - & - & - & Paleártica occidental \\
\hline \multicolumn{11}{|l|}{ Protoribatidae J. y P. Balogh, 1984} \\
\hline Protoribates (P.) capucinus Berlese, 1908 & - & - & - & $\mathrm{x}$ & - & - & - & - & - & Cosmopolita \\
\hline Protoribates (Triaungius) obtusus (Mihelčič, 1956) & - & - & - & - & $\mathrm{x}$ & - & - & - & - & Paleártica meridional \\
\hline \multicolumn{11}{|l|}{ Haplozetidae Grandjean, 1936} \\
\hline Peloribates tunisiensis Mahunka, 1980 & - & - & $\mathrm{X}$ & - & - & $\mathrm{x}$ & $\mathrm{x}$ & - & - & Mediterránea occidental \\
\hline Pilobates carpetanus Pérez-İ̃nigo, 1969 & - & - & - & - & - & - & $\mathrm{x}$ & - & - & Mediterránea \\
\hline \multicolumn{11}{|l|}{ Galumnidae Jacot, 1925} \\
\hline Allogalumna parva (Berlese, 1916) & - & $x$ & - & - & - & - & - & - & - & Mediterránea \\
\hline Cryptogalumna cryptodonta Grandjean, 1957 & - & - & - & $\mathrm{X}$ & - & - & $\mathrm{X}$ & - & - & Mediterránea occidental \\
\hline Galumna gibbula Grandjean, 1956 & - & $x$ & $\mathrm{x}$ & - & $\mathrm{x}$ & $x$ & - & $\mathrm{x}$ & - & Mediterránea \\
\hline Galumna lanceata (Oudemans, 1900) & - & - & - & - & - & - & - & - & - & Paleártica, Vietnam \\
\hline Galumna tarsipennata Oudemans, 1914 & - & - & - & - & - & - & - & $x$ & - & Paleártica meridional \\
\hline Pergalumna myrmophila (Berlese, 1914) & - & - & $x$ & - & - & $x$ & $\mathrm{X}$ & $x$ & - & Paleártica meridional \\
\hline
\end{tabular}

